Reprod. Nutr. Develop., 1988, 28 (3 B), 845-856

\title{
Différenciation du tissu musculaire. Conséquences technologiques pour la filière viande
}

C. VALIN

Département de Technologie de la Viande. I.N.R.A., Theix, 63122 Ceyrat, France.

Summary. Muscle differentiation. Technological consequences for the meat industry.

The post-mortem changes of muscle into meat is based on biochemical processes which affect muscle structure and composition. The first step in the conversion of muscle into meat is the development of rigor mortis which begins as ATP is depleted. When there is insufficient ATP in the muscle, actin and myosin filaments together induce the muscle stiffing. The rigidity of the muscle, which makes the meat very tough, is not infinitely maintained. Hence, the muscle softens again during the so called ageing period, partly caused by a proteolytic disruption of the cross bridged myofibrillar proteins. The extent and intensity of these changes are determined primarily by muscle typing which defines the amount of substrates and nature of enzyme systems involved in these reactions and secondly by the post-mortem handling procedures in particular the chilling conditions.

There is a large variability of muscle types within a carcass according to their location and function and similar muscles in different animals also exhibit a large variability of types. This explains the great qualitative differences observed in the organoleptic (colour, tenderness and flavour) and functional (water holding capacity, emulsifying properties) properties of meat. Whatever the species, muscle type is a dynamic muscle characteristic affected by age, sex, growth rare and hence by the animal production system used. Thus, to fit the requirements for high quality meat or to optimize the meat price/quality ratio, the quantitative effects of differences in muscle typing on the variability of meat characteristics should be further investigated.

\section{Introduction.}

La transformation du muscle en viande repose très largement sur des mécanismes biochimiques qui, après la mort des animaux, modifient plus ou moins profondément la composition et la structure du muscle. Les réactions mises en jeu, essentiellement des réactions hydrolytiques, dissipent dans un premier temps les réserves énergétiques du muscle (ATP, PC, glycogène) au cours de l'installation de la rigor mortis, puis affectent l'organisation des protéines musculaires de structure au cours de la phase dite de maturation. La cinétique et l'intensité de ces réactions définissent en dernier ressort les qualités technologiques, organoleptiques, hygiéniques et nutritionnelles des viandes. 
Différents facteurs influent sur le cours de ces transformations : des facteurs intrinsèques, au muscle, son typage, qui définit la composition et la nature des équipements enzymatiques impliqués dans ces transformations, mais également l'âge, le sexe, et l'espèce qui ne sont pas sans effet sur les caractéristiques de typage des muscles, des facteurs extrinsèques, à savoir les technologies mises en $œ u v r e$ et en particulier le régime thermique imposé aux carcasses et aux viandes.

Chez un animal de boucherie les muscles présentent une grande diversité de types métaboliques et contractiles selon leur localisation et leur fonction. On observe également pour un muscle donné des différences de typage notables entre animaux. L'ensemble des ces variations sous-tend une large part de la variabilité des caractéristiques qualitatives des viandes.

S'il est permis de penser que nous pourrons influencer dans le futur les types musculaires chez les animaux de boucherie, soit par la sélection, soit par des traitements infléchissant la différenciation et le développement musculaire, il est fondamental tout d'abord de mieux connaître les relations typage-qualités des viandes afin de pouvoir apprécier les répercussions possibles de telles manipulations. Les quelques exemples que nous présentons montrent le poids du typage musculaire dans la définition des caractéristiques des viandes mais révèlent également l'étendue des problèmes non résolus, compte tenu en particulier de la diversité des objectifs visés.

\section{Influence des caractéristiques contractiles et métaboliques des muscles sur le mécanisme d'installation de la rigor mortis.}

\subsection{Schéma général de la rigor mortis.}

La première phase des transformations post-mortem du muscle en viande est caractérisée au plan biochimique par le catabolisme des composés musculaires riches en énergie, ATP et PC. La disparition de I'ATP qui s'accompagne d'une chute du $\mathrm{pH}$ musculaire, entraîne une modification profonde des propriétés du tissu musculaire qui perd son élasticité et devient rigide.

Le schéma général du catabolisme post-mortem de l'ATP a été proposé dès 1973 par J. R. Bendall. Après l'abattage des animaux, l'ATP est constamment et lentement hydrolysé dans le tissu musculaire selon une réaction du type :

$\mathrm{MgATP}^{-2}+\mathrm{H}_{2} \mathrm{O} \stackrel{\text { ATPases }}{\longrightarrow} \mathrm{MgADP}^{-1}+\mathrm{Pi}^{-2}+\mathrm{H}^{+}$

Cette réaction libère un proton et l'ADP libéré peut être rephosphorylé soit par la phosphocréatine (PC)

$\mathrm{MgADP}^{-1}+\mathrm{PC}^{-2}+\mathrm{H}^{+} \stackrel{\text { créatine kinase }}{\longrightarrow} \mathrm{MgATP}^{-2}+$ créatine

avec consommation d'un proton, soit par la voie glycolytique activée par la libération de $\mathrm{Pi}^{-2}$ lors de la réaction [1] avec rephosphorylation de 3 ADP pour une unité glucose consommée selon [3].

$3 \mathrm{MgADP}^{-1}+3 \mathrm{Pi}^{-2}+1$ glucose $+1 \mathrm{H}^{+} \rightarrow 3 \mathrm{MgATP}^{-2}+2$ lactate $^{-1}$ 
La resynthèse d'ATP par la voie glycolytique est consommatrice de protons. Mais quand I'ADP substrat provient de l'hydrolyse de l'ATP le résultat net est une acidification ( $2 \mathrm{H}+$ /glucose).

Le bilan net peut s'écrire :

(glucose $)_{n}+\mathrm{PC}^{-2}+2 \mathrm{H}_{2} \mathrm{O} \rightarrow$ créatine $+\mathrm{Pi}^{-2}+2$ lactate $^{-1}+2 \mathrm{H}^{+}+$ $(\text { glucose })_{n-1}$

Post-mortem le renouvellement de l'ATP sera assuré tant que les réserves de $\mathrm{PC}$ et glycogène le permettront et que la chute de $\mathrm{pH}$ n'inhibera pas la voie glycolytique. Globalement le turnover des liaisons phosphates riches en énergie peut être évalué selon Bendall (1973) à l'aide de la relation :

$\mathrm{V} \sim \sim_{\mathrm{P}}=\mathrm{V}_{\mathrm{ATP}}+\mathrm{V}_{\mathrm{PC}}+1,5 \beta \cdot \mathrm{V}_{\mathrm{pH}}$

où $V_{A T P}$ et $V_{P C}$ sont les vitesses d'hydrolyse de I'ATP et de PC, $\mathrm{VpH}$ la vitesse de chute du $\mathrm{pH}$ et $\beta$ le pouvoir tampon du muscle.

Les principales conséquences de ce schéma sont les suivantes:

- I'acidification du muscle post-mortem est due au turnover de l'ATP ;

- la vitesse d'acidification est fonction de la vitesse de turnover de l'ATP ;

- l'amplitude de la chute de $\mathrm{pH}$ dépend de l'importance des réserves énergétiques ( $P C$, glycogène) susceptibles d'entretenir ce turnover post-mortem.

L'étude à l'aide d'une méthode non invasive, en l'occurrence la $R M N$ du ${ }^{31} p$ a permis de suivre en continu ces transformations, d'en quantifier les différents paramètres (Renou et al., 1986) et de vérifier l'hypothèse émise par Bendall en 1973.

\subsection{Relations entre les types métabolique et contractile des fibres et l'installation de la rigor mortis.}

\subsection{Typage et réserves énergétiques.}

a) Composés phosphorylés. - II existe une relation entre le typage des muscles et leurs teneurs en composés phosphorylés. Dans le cas du lapin la comparaison de trois muscles types, $m$. psoas pure $\alpha W$, dans cette espèce, le $m$. gastrocnémien median, mélange en proportions identiques de fibres $\alpha W, \alpha R$ et $\beta \mathrm{R}$, et le $\mathrm{m}$. conoïde pure $\beta \mathrm{R}$ chez le lapin adulte, permet d'observer des teneurs comparables dans les deux muscles rapides, mais très supérieures à celles mesurées dans le conoïde (tabl. 1).

Le muscle conoïde contient par ailleurs un composé phosphorylé le glycérophosphoryl choline (environ $18 \mu \mathrm{mol} . \mathrm{g}$ ) non mesurable dans les deux autres muscles. Une étude complémentaire de ce composé dans les muscles d'agneau confirme sa présence dans les muscles très riches en fibres $\beta \mathrm{R}$ (Renou et al., 1986). Le rôle du GPC dans le muscle demeure inexpliqué.

b) Glycogène. - La relation type des fibres teneur en glycogène a été l'objet de nombreuses controverses, la confusion tenant au fait que de nombreux 
TABLEAU 1

Concentration en composés phosphorylés des muscles de lapin.

\begin{tabular}{rccr}
\hline & M. Psoas & $\begin{array}{c}\text { M. Gastrocnemius } \\
(\mu \mathrm{mol} / \mathrm{g} \text { de tissu frais })\end{array}$ & M. Conoïde \\
\hline ATP & 8,7 & 7 & 3,8 \\
PC & 23 & 21 & 6,9 \\
Pi & 11 & 12,5 & 19,0 \\
SP & 8 & 5,4 & 4,9 \\
GPC & 0 & 0 & 14,1 \\
TP & 50,7 & 45,9 & 48,7 \\
\hline
\end{tabular}

TP : composés phosphorylés totaux détectables par RMN du ${ }^{31} \mathrm{P}$; $\mathrm{SP}$ : sucres phosphates ; GPC : glycérophosphorylcholine; $\mathrm{Pi}$ : phosphate; $\mathrm{PC}$ : phosphocréatine.

auteurs ne faisaient pas de distinction entre fibres rouges rapides et lentes. Peter et al., (1972) montraient chez le cobaye qu'en fait les plus hautes teneurs en glycogène s'observaient dans les muscles rouges rapides et les plus faibles dans les rouges lents. Chez les bovins la différence est moins marquée mais la tendance est similaire, à l'exception du muscle diaphragme, et on observe une relation inverse entre la valeur du pH ultime et l'activité ATPasique myofibrillaire (Monin, 1980).

Mais le typage peut également influer sur la teneur en glycogène à l'abattage par le biais de la variabilité qu'il introduit dans la vitesse de mobilisation du glycogène lors des stress ante abattage que les animaux peuvent subir. Dans le cas de stress de groupe, par exemple, la mobilisation du glycogène est beaucoup plus rapide dans les fibres de type $\alpha$ que dans les fibres lentes (Lacourt et Tarrant, 1981). Ce résultat est tout à fait cohérent avec les différences de $\mathrm{pH}$ ultime observées entre muscles d'une même carcasse lorsque les animaux sont soumis à des stress importants (Tarrant et Sherington, 1980), les muscles rapides de l'arrière étant les plus affectés.

\subsection{Typage et cinétique du turnover de l'ATP post-mortem.}

Toutes choses égales par ailleurs et en particulier la température, le typage influe sur la cinétique du turnover post-mortem de l'ATP comme l'illustrent les données du tableau 2 obtenues sur lapin à $25^{\circ} \mathrm{C}$ (Renou et al., 1986).

Dans la phase initiale au cours de laquelle la concentration en ATP est constante, les mesures de la vitesse d'apparition de Pi constitue une mesure de l'activité ATPasique qui apparaît naturellement plus élevée dans le muscle rapide. Mais la teneur initiale en PC est beaucoup plus faible dans le muscle lent, ce qui conduit à observer une plus courte période de resynthèse de I'ATP et finalement une rigor plus rapide dans le muscle le plus lent.

La vitesse d'acidification n'apparaît pas très différente entre les deux types de muscle. Elle est de l'ordre de $3.10^{-3}$ unité $\mathrm{pH} / \mathrm{min}$ dans les deux cas alors que la glycolyse est plus efficiente dans les muscles de type rapide que dans les muscles 
TABLEAU 2

Valeurs initiales et vitesses de transformation post mortem des composés phosphorylés des muscles de lapin. Installation de la rigor mortis à $25^{\circ} \mathrm{C}$.

\begin{tabular}{|c|c|c|c|}
\hline & M. Psoas & M. Gastrocnemius & M. Conoïde \\
\hline $\begin{array}{l}\mathrm{Pi} \\
{[\mathrm{Pi}]_{0}(\mu \mathrm{mol} / \mathrm{g})}\end{array}$ & 4 & 13 & 11,5 \\
\hline Ratex $10^{-3}(\mu \mathrm{mol} / \mathrm{g} / \mathrm{min})$ & 113 & 57 & 30 \\
\hline $\begin{array}{l}\mathrm{PC} \\
{[\mathrm{PC}]_{0}(\mu \mathrm{mol} / \mathrm{g})}\end{array}$ & 38 & 21 & 8.7 \\
\hline Ratex $10^{-3}(\mu \mathrm{mol} / \mathrm{g} / \mathrm{min})$ & 9,8 & 13,6 & 25,2 \\
\hline $\begin{array}{l}\text { ATP } \\
{[\text { ATP }]_{0}(\mu \mathrm{mol} / \mathrm{g})}\end{array}$ & 8,7 & 7 & 3,8 \\
\hline $\begin{array}{l}\mathrm{t}_{1 / 2}(\mathrm{~min}) \\
\mathrm{TP}(\mu \mathrm{mol} / \mathrm{g})\end{array}$ & $\begin{array}{l}326 \\
50,7\end{array}$ & $\begin{array}{r}300 \\
41\end{array}$ & $\begin{array}{l}240 \\
42,3^{a}\end{array}$ \\
\hline
\end{tabular}

TP : composés phosphorylés totaux détectables par RMN du ${ }^{31} \mathrm{P}$; TPa: composés phosphorylés totaux détectables par RMN du ${ }^{31} \mathrm{P}$ en prenant en compte une teneur initiale de ce muscle

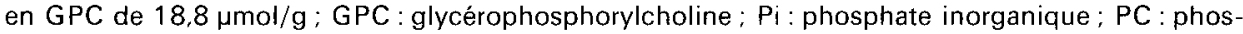
phocréatine.

de type lent. II est vraisemblable que les différences de pouvoir tampon entre les deux types de fibres expliquent ces vitesses d'acidification comparables entre les deux types extrêmes de muscles étudiés. Dans le cas des agneaux, entre muscles à prédominance de fibres de type rapide comme le tensor fasciae latae et à prédominance de fibres de type lent comme le supraspinatus, on observe des différences importantes de pouvoir tampon (Houlier et al., 1984).

Dans des conditions de réfrigération rapide pouvant induire le phénomène de contraction au froid, I'hétérogénéité de la réponse des muscles est également imputable à leur typage. En particulier les muscles lents, dont l'activité ATPasique myofibrillaire est inhibée aux températures inférieures à $+10^{\circ} \mathrm{C}$, ne présentent pas le phénomène de la contraction au froid qui se développe essentiellement dans les muscles riches en fibres rouges rapides (Bendall, 1973). Pour ces derniers l'inhibition de la contraction au froid ne peut être obtenue que si le $\mathrm{pH}$ est inférieur à 6,0 , ce qui a conduit, au plan industriel, à la mise au point de la technique de stimulation électrique.

\subsection{Implications technologiques.}

Du fait de sa masse la carcasse ne se refroidit pas d'une façon homogène. Dans le cas des bovins en particulier, les gradients de température qui s'établissent lors de la réfrigération provoquent une hétérogénéité de vitesse de chute du $\mathrm{pH}$ qui peut expliquer jusqu'à $90 \%$ de la variabilité observée entre muscles d'une même carcasse, ce qui atténue les effets du typage. Cependant un désossage précoce après l'abattage redonne à ce dernier facteur un poids important. De plus, dans le but d'accélérer l'installation de la rigor mortis certains traitements 
technologiques peuvent être appliqués dans les instants qui suivent l'abattage, donc dans un milieu relativement homogène au plan de la température; c'est le cas de la stimulation électrique, et dans ces conditions le typage a un effet très significatif sur l'efficacité de ce traitement.

Une étude des facteurs de variation de l'efficacité de la stimulation électrique met bien en évidence l'importance du typage.

La comparaison de trois muscles caractéristiques des classes, rouge lent, le supra spinatus, rouge rapide, le longissimus dorsi, et blanc rapide, le tensor du fasciae latae révèle les interactions existant entre les paramètres de la stimulation, temps d'application et intensité du champ électrique et les caractéristiques du typage sur l'efficacité du process (tabl. 3).

\section{TABLEAU 3}

Influence des conditions de stimulation et du typage sur la vitesse de turnover de l'ATP en cours de stimulation (Houlier et al., 1984).

\begin{tabular}{llcccr}
\hline Sources de variation & \multicolumn{5}{c}{ Valeurs moyennes } \\
\hline 1. Durée de stimulation & 0,922 & 0,610 & 0,336 & 0,223 & $173,0^{* *}$ \\
2. Typage & 0,670 & 0,517 & 0,380 & & $49,9^{* *}$ \\
3. Champ électrique & 0,436 & 0,517 & 0,548 & 0,590 & $7,6^{* *}$ \\
Interactions 1-2 & & & & & $9,2^{* *}$ \\
Interactions 1-3 & & & & 0,8 NS \\
Interactions 2-3 & & & & & $1,93 \mathrm{NS}$ \\
\hline
\end{tabular}

Donc le typage des muscles affecte directement l'efficacité de la stimulation électrique. En particulier, les muscles rapides sont stimulés efficacement à bas champ $(1,5-2 \mathrm{~V} / \mathrm{cm})$ alors que les muscles lents nécessitent l'application d'un champ élevé $(3-4 \mathrm{~V} / \mathrm{cm})$ pendant un temps relativement long, 1 à $2 \mathrm{~min}$. Ces dernières conditions sont difficiles à mettre en œuvre dans la pratique industrielle qui impose des temps de traitement courts. Sur ces bases on peut espérer optimiser le process de stimulation mais la variabilité inter muscles due au typage ne permettra pas d'obtenir un effet homogène intra carcasse dudit traitement.

\section{Maturation et attendrissage post-mortem de la viande.}

\subsection{Schéma général de la maturation.}

La maturation est une phase de l'évolution post-mortem du muscle également très importante mais moins bien connue que la rigor mortis. C'est au cours de la maturation qu'interviennent des modifications de la texture du muscle qui font que la viande est plus ou moins tendre. Ces modifications sont importantes par leur ampleur puisque la résistance mécanique de la viande bovine après cuisson est diminuée d'un facteur 4 par la maturation, pratiquement quel que soit le muscle, et la résistance myofibrillaire propre par le même facteur. La texture de la viande est définie par l'état et l'organisation des protéines de structure du muscle, le collagène, les protéines myofibrillaires et du cytosquelette. Au cours de 
la maturation l'attendrissage est dû à des modifications des myofibrilles et du cytosquelette. Par contre la maturation a peu d'effet sur les propriétés thermiques et mécaniques du collagène. L'évolution de la structure myofibrillaire est pour une large part consécutive à une attaque protéolytique vraisemblablement par deux groupes de protéases musculaires, les protéinases neutres activées par le calcium et les protéinases lysosomales. S'agissant d'un processus enzymatique, sa vitesse est fonction de la température. Entre 0 et $40^{\circ} \mathrm{C}$, le coefficient de température est très élevé $\left(Q_{10}=2,4\right)$ (Davey et Gilbert, 1976).

\subsection{Les facteurs de variation de l'efficacité de la maturation.}

\subsection{L'effet température.}

L'analyse des mécanismes de la maturation a été rendue difficile du fait de l'absence jusqu'à une période très récente de méthodes quantitatives d'appréciation des modifications des protéines de structure et de leur impact sur la texture de la viande. A partir des mesures de résistance mécanique, dans le cas des muscles de bovins, Dransfield et al., (1981) ont pu analyser la cinétique de maturation dans une plage de température allant de 0 à $20^{\circ} \mathrm{C}$ et montrer que dans ces conditions la température était le facteur majeur de variation de la vitesse d'attendrissage, 32 fois plus important selon ces auteurs que l'effet animal, 10 fois plus élevé que I'effet muscle.

A l'inverse de la rigor mortis, dans la pratique, le facteur température est peu susceptible de variation puisque les contraintes d'hygiène impliquent de conserver la viande à basse température. Dans ces conditions, en analysant quantitativement l'évolution de la structure myofibrillaire à l'aide d'une technique biochimique (Ouali, 1981), on observe un effet muscle majeur sur la variabilité de l'efficacité de ces transformations, et un effet animal du même ordre de grandeur que la variation imputable au temps de conservation.

\subsection{L'effet muscle.}

Une étude de Gann et Merkel (1978) a révélé dans le cas du muscle long dorsal de bovin qu'au niveau des fibres musculaires, les fibres de type rapide maturaient plus rapidement que les fibres de type lent (destruction des stries $Z$, de l'alignement des sarcomères, coupures dans les myofilaments). Cette observation a été étendue aux autres espèces. Sur cette base, qui suggère une relation entre le typage des fibres et le mécanisme de la maturation, on est conduit à examiner comment le typage influe sur les substrats et les enzymes impliqués dans cette transformation post-mortem.

Au niveau des substrats, on sait encore peu de choses mais il a toutefois été rapporté que les myofibrilles de type lent sont moins sensibles à la protéolyse que celles de type rapide, du moins dans le cas de l'attaque in vitro de cette structure par la protéase neutre activée par le calcium (Ouali et al., 1983). D'ailleurs les myosines lentes et rapides montrent également des susceptibilités différentes à la 
protéolyse tant par les protéases neutres que par les protéases lysosomales (Dufour et al., 1987), différences du même type que celles enregistrées au niveau des myofibrilles. En ce qui concerne les enzymes, il apparaît de plus en plus sûrement une localisation en fonction du type de fibre. Différents auteurs ont noté une teneur plus élevée en protéases dans les muscles rouges. Mais jusqu'à présent une appréciation quantitative de l'équipement protéolytique était très difficile, en particulier pour les protéases lysosomiales, du fait de l'existence d'inhibiteurs tissulaires qui rendent cette mesure très aléatoire. Cependant les premiers résultats obtenus au laboratoire fondés sur l'utilisation de sondes moléculaires, indiquent en particulier chez le bovin, la présence d'une quantité beaucoup plus élevée de cathepsine $B$ et $D$ dans le muscle LD de type prédominant $\alpha R$ que dans le diaphragme qui comprend une majorité de fibre $\beta \mathrm{R}$. Ce résultat tendrait à confirmer l'existence d'une répartition dépendante du type. II en va d'ailleurs de même pour les inhibiteurs sarcoplasmiques des cystéines protéinases lysosomales de poids moléculaire élevé (Ouali et al., 1986) beaucoup plus abondants dans les fibres rouges lentes.

\subsection{Analyse de l'effet muscle sur la vitesse de maturation : cas des bovins.}

Sur une population de bovins de types très différents par la race, le sexe et l'âge nous avons testé l'existence d'une relation entre la vitesse de maturation mesurée biochimiquement (Ouali, 1981) et les caractéristiques contractile et métabolique déterminées par des mesures d'activités enzymatiques (Talmant et al., 1986) et ceci sur huit des principaux muscles de la carcasse.

Tous animaux et tous muscles confondus, une régression linéaire multiple permet d'expliquer $36 \%$ de la variabilité de la vitesse de maturation avec un coefficient de corrélation multiple hautement significatif $(P<0,001)$. On constate que la vitesse de maturation est d'autant plus élevée que le muscle est plus rapide (activités ATPasique et LDH), et moins rouge (teneur en fer héminique et activité citrate synthase) et que le $\mathrm{pH}$ ultime atteint au cours de la rigor mortis est plus bas.

Pour préciser la relation on peut scinder les muscles étudiés en deux groupes. Un groupe de muscles qui maturent rapidement ( $L D, T F L, S M$ ) et un groupe de muscles qui maturent plus lentement (RA, TB, PP). le premier groupe rassemble d'ailleurs des muscles de type plus rapide et moins rouge que le second. Pour les deux groupes, on constate une variabilité de la vitesse de maturation. Pour le premier, la vitesse de maturation est d'autant plus élevée qu'ils sont plus rapides alors que pour le second groupe leur vitesse de maturation est d'autant plus élevée qu'il sont également plus rapides, mais aussi moins rouges, et que leur $\mathrm{pH}$ ultime est plus bas, ce dernier facteur étant le plus discriminant.

L'ensemble de ces résultats indique un effet significatif du typage sur la vitesse de maturation mais le pourcentage de variabilité expliqué est tout de même limité, ce qui peut signifier également que le typage est mal apprécié par les mesures mises en cuvre.

Sur l'ensemble des résultats obtenus une analyse en composante principale indique d'ailleurs que les paramètres mesurés, vitesse de maturation comprise, permettent une meilleure discrimination des animaux que des muscles. 
L'examen des variables les plus discriminantes de l'effet muscle et de l'effet animal est résumé dans le tableau 4.

TABLEAU 4

Pouvoir discriminant des différentes variables mesurées caractérisant les effets muscle et animal.

\begin{tabular}{lcr}
\hline & \multicolumn{3}{c}{$\begin{array}{c}\text { Pouvoir discriminant } \\
\text { (valeur du F) }\end{array}$} \\
\cline { 2 - 3 } Variables & Effet muscle & Effet animal \\
\hline Vitesse maturation & $4,15^{* *}$ & $4,45^{* * *}$ \\
ATPase & $3,74^{* *}$ & $5,46^{* * *}$ \\
LDH & $2,50^{*}$ & $7,96^{* * *}$ \\
Citrate synthase & $8,54^{* * *}$ & $2,47^{*}$ \\
Fer héminique & $2,21^{*}$ & $12,93^{* * *}$ \\
dpH & $6,23^{* * *}$ & $5,90^{* * *}$ \\
\hline
\end{tabular}

${ }^{*} \mathrm{P}<0,5 ;{ }^{* *} \mathrm{P}<0,1 ;{ }^{* * *} \mathrm{P}<0,001$.

Les variables utilisées pour apprécier le typage contractile et métabolique sont plus influencées par le type de l'animal que par le muscle. Par contre, vitesse de maturation et amplitude de chute du $\mathrm{pH}$ sont plus typiques de l'effet muscle en restant néanmoins très tributaires des animaux.

Les résultats enregistrés dans cette expérimentation posent le problème de la signification du typage des muscles lorsque l'on considère une population aussi hétérogène que celle testée.

\section{La couleur.}

L'évolution des modes de distribution des viandes sous forme de portions consommateurs préemballées conservées à l'état réfrigéré redonne de l'importance au facteur couleur qui est déterminant dans l'acte d'achat puisque maintenant c'est très largement le consommateur qui se sert lui-même. Pour l'économie du système de distribution il est donc très important que la couleur de la viande soit stable. La dégradation de la couleur est liée à l'oxydation progressive de la myoglobine en metmyoglobine de teinte marron alors que la teinte rouge de la viande est due à la présence en surface de myoglobine oxygénée rouge vif plus ou moins mélangée selon les conditions de conservation avec de la myoglobine réduite rouge sombre.

La stabilité de la couleur dépend des vitesses relatives d'autoxydation du pigment et de réduction de la metmyoglobine, le muscle possédant des systèmes réducteurs susceptibles post-mortem de réduire la metmyoglobine tant que des équivalents réducteurs sont disponibles (NADH). En dehors des conditions de conservation qui peuvent ou non favoriser la stabilité de la couleur, il existe une variabilité intermusculaire importante (Hood, 1983, tabl. 5).

Les résultats de Renerre et Labas, (1987) obtenus sur muscles de bovin permettent de préciser les données de Hood. Ce sont les musles à vitesse de consommation de l'oxygène élevée post-mortem qui sont les moins stables. Ces 
mêmes muscles ont également une vitesse de réduction élevée de la metmyoglobine, ce qui conduit à considérer que le facteur dominant qui règle la stabilité de la couleur est la vitesse d'autoxydation du pigment. Un pourcentage élevé de fibres rouges lentes n'est pas favorable à une bonne stabilité de la couleur.

TABLEAU 5

Facteurs de variabilité de la stabilité de la couleur (Hood, 1983).

\begin{tabular}{lcc}
\hline Effet & \% de la variabilité & F-signification \\
\hline Animal & 7,2 & $\mathrm{p}<0,001$ \\
Muscle & 45,5 & $\mathrm{p}<0,001$ \\
Température & 32,5 & $\mathrm{p}<0,001$ \\
Muscle-température & 3,5 & $\mathrm{p}<0,05$ \\
Résiduel & 12,0 & \\
Total & 100 & \\
\hline
\end{tabular}

\section{La flaveur.}

Que les viandes rouges, c'est-à-dire riches en pigment mais également en lipides intramusculaires, présentent une intensité de flaveur supérieure à celle des viandes blanches est un fait bien connu et vérifié pour les viandes des animaux des différentes espèces bouchères.

Si on examine plus précisément la relation composition/qualité organoleptique, et plus spécifiquement la relation teneur en lipides et intensité de la flaveur on constate que l'effet améliorateur de la teneur en lipides est vite plafonné. Dans le cas des viandes bovines, (Smith et al., 1983) ont montré dans une étude portant sur plusieurs milliers d'animaux, qu'au-delà d'une teneur en lipides intramusculaires de 3,5\% dans le muscle long dorsal, l'intensité de la flaveur n'était pas améliorée voire régressait. Dans le cas des viandes de porc un résultat tout à fait analogue a été obtenu par les chercheurs danois.

Dans une étude antérieure (Goutefongea et Valin, 1978) et lors d'observations similaires nous avions été amenés à émettre I'hypothèse que la relation lipide/flaveur pouvait en fait traduire un effet du type métabolique. Nous avons vérifié cette hypothèse à l'aide d'une expérience réalisée sur agneaux (Valin et al., 1982) et montré d'une façon directe que l'intensité de flaveur d'un muscle donné est fonction de son typage et, par la même occasion, qu'une faible variation de typage, en l'occurrence apprécié par les techniques histochimiques classiques, pouvait entraîner des différences hautement significatives des qualités organoleptiques et plus précisément de la jutosité et de la flaveur.

\section{Conclusion.}

Les quelques exemples rapportés, sans constituer une liste exhaustive des relations connues entre typage des muscles et qualités de la viande montrent que cette relation existe et affecte très directement les mécanimes biochimiques qui, post-mortem assurent la transformation du muscle en viande, installation de la rigor mortis, évolution de la texture et de la couleur en cours de conservation. 
Le typage des muscles qui définit leur composition, influence donc également des caractéristiques qualitatives telles que la couleur, par le biais de la teneur en pigment, mais aussi la flaveur et vraisemblablement aussi des caractéristiques technologiques telle que la rétention d'eau et le pouvoir émulsifiant compte tenu des différences de $\mathrm{pH}$ ultime et des différences observées d'hydrophobicité liées au polymorphisme des protéines musculaires.

Enfin le troisième point qui mérite d'être souligné se rapporte aux effets très significatifs que de faibles variations de typage d'un muscle donné entraînent au plan des qualités organoleptiques, la flaveur étant un exemple particulièrement démonstratif.

Le typage des muscles est une caractéristique dynamique qui est affectée par l'âge des animaux, le sexe, la vitesse de croissance donc par les modes de production mis en œuvre. Dans l'état actuel des connaissances, on ne sait pas réellement quantifier les effets de ces variations sur la qualité de la viande, pour plusieurs raisons qui tiennent autant à une approche par trop simplificatrice du typage des muscles qu'à un manque de méthodes quantitatives d'appréciation des caractéristiques organoleptiques des viandes. II faudra impérativement atteindre cette quantification du poids du typage sur les différentes caractéristiques qualitatives des viandes avant de mettre en œuvre, au niveau des productions animales, des manipulations susceptibles d'affecter profondément les caractéristiques musculaires des animaux de boucherie.

$13^{e}$ Réunion du groupe Développement I.N.R.A. Cap d'Agde, 25-27 mai 1987.

\section{Références}

BENDALL J. R., 1973. Post-mortem changes in muscle. Bourne G. H., The structure and function of muscle. 2nd Ed., 2, 244-309.

DAVEY C. L., GILBERT K. V., 1976. The temperature coefficient of beef aging. J. Sci Food Agric., 27, 244-250.

DUFOUR E., OUALI A., OBLED A., DEVAL C., VALIN C., 1987. Lysosomal proteinases sensitive regions in fast and slow skeletal muscle myosin. Arch. Bioch. Bioph. (in press).

DRANSFIELD E., JONES C. R. D., MAC FIE H.J.H., 1981. Quantifying changes in tenderness during storage of beef. Meat Sci., 5, 131-137.

GANN G. L., MERKEL R. A., 1978. Ultrastructural changes in bovine longissimus dorsi muscle during post-mortem ageing. Meat Sci., 2, 119-144.

GOUTEFONGEA R., VALIN C., 1978. Etude de la qualité des viandes bovines : comparaison des qualités organoleptiques des viandes de taurillon et d'animal adulte. Ann. Tech. agric., 27, 609-627.

HOOD T., 1983. The chemistry of Vacuum and gas packaging of meat. In Recent Advances in the Chemistry of meat, 11, 213-230. The Royal Society of Chemistry, Burlington House, London.

HOULIER B., VALIN C., MONIN G., SALE P., 1984. Effect of muscle type on electrical stimulation efficiency. Sci. Alim., 4, 167-175.

LACOURT A., TARRANT P. V., 1981. Selective glycogen depletion and recovery in skeletal muscles fibre types of young bulls subjected to a behavioural stress, 417-429. In The problem of dark cutting in beef. Martinus Nijhoff Publ. 
MONIN G., 1981. Muscle metabolic type and the DFD condition, 63-81. In The problem of dark cutting in beef. Martinus Nijhoff Publ.

OUALI A., 1981. Variation entre muscles de l'effet de la maturation sur l'activité ATPasique des myofibrilles. Sci. Alim., 1, 1-6.

OUALI A., DUFOUR E., OBLED C., DEVAL C., VALIN C., 1988. Action des protéinases musculaires sur les myosines rapide et lente. Relation avec la protéolyse post-mortem dans des muscles de type contractile variable. Reprod. Nutr. Dev., 28, (in press).

OUALI A., OBLED A., COTIN P., MERDACI N., DUCASTAING A., VALIN C., 1983. Comparative effects of post-mortem storage and low Ca requiring neutral proteinase on bovine and rabbit myofibrillar proteins. J. Sci. Food Agric., 34, 466-476.

OUALI A., BIGE L., OBLED A., LACOURT A., VALIN C., 1986. Small and high molecular weight proteinase inhibitors from bovine muscle in cysteine proteinases and their inhibitors, 545-554. In Cysteine proteinases and their inhibitors. Walter de Gruyter and Co, Berlin.

PETER J. B., BARNARD R. J., EDGERTON V. R., GILLEPSIE C. A., STEMPAL G. E., 1972. Metabolic profiles of three fibre types of skeletal muscles in guinea pigs and rabbits. Biochemistry, 11, 2627-2633.

RENERRE M., LABAS R., 1987. Biochemical factors influencing metmyoglobin formation in beef muscle. Meat Sci. 19, $151-165$.

RENOU J.P., CANIONI P., GATELLIER Ph., VALIN C., COZZONE P. J., 1986. Phosphorus 31 nuclear magnetic resonance study of post-mortem catabolism and intracellular $\mathrm{pH}$ in intact excised rabbit muscle. Biochimie, 68, 543-554.

SMITH G. C., SAVELL J. W., CROSS H. R., CARPENTER Z. L., 1983. The relationship of USDA quality grade to beef flavor. Food Technology, 5, 233-238.

TALMANT A., MONIN G., BRIAND M., DADET M., BRIAND Y., 1986. Activities of metabolic and contractile enzyme in 18 bovine muscle. Meat Sci., 18, 23-40.

TARRANT P. V., SHERINGTON J., 1980. An investigation of ultimate $\mathrm{pH}$ in the muscle of commercial beef carcasses. Meat Sci., 4, 287-298.

VALIN C., TOURAILLE C., VIGNERON P., ASHMORE C. R., 1982. Prediction of lamb meat quality traits based on muscle biopsy fiber typing. Meat Sci, 6, 257-263. 